\title{
Diabetes y enfermedad cardiovascular, aspectos actuales de su manejo: la visión del cardiólogo
}

\author{
Diabetes and cardiovascular disease, current aspects of its management: \\ the cardiologist's vision
}

\author{
Ramírez-Ramos $C F,{ }^{1}$ Betancur $K,{ }^{1}$ Castilla $G A,{ }^{2}$ Saldarriaga $S,{ }^{2}$ Aranzazu-Uribe $M,{ }^{2}$ \\ Ramírez-Méndez DA, ${ }^{3}$ Herrera-Céspedes $E,{ }^{4}$ Salamanca-Montilla JF, ${ }^{4}$ Martínez CA, ${ }^{1}$ Saldarriaga $C^{5}$
}

\begin{abstract}
${ }^{1}$ Departamento de cardiología, Universidad Pontificia Bolivariana, Clínica CardioVID, Medellín, Colombia.

${ }^{2}$ Departamento de medicina interna, Universidad Pontificia Bolivariana, Medellín, Colombia.

${ }^{3}$ Médico, Universidad Surcolombiana, Medellín, Colombia. 4Departamento de medicina interna, Hospital Universitario Hernando Moncaleano y Universidad Surcolombiana, Neiva, Colombia.

${ }^{5}$ Departamento de cardiología e insuficiencia cardíaca, Universidad Pontificia Bolivariana, Universidad de Antioquia y Clínica CardioVID, Medellín, Colombia.
\end{abstract}

Autor de correspondencia: Cristhian Ramírez, MD Correo electrónico: cristhianramos1989@hotmail.com Fecha de recepción: 17/02/2020

Fecha de aceptación: 6/05/2020

\section{Resumen}

La diabetes es una enfermedad prevalente, con casos en aumento en todo el mundo, y se relaciona de forma directa con otras condiciones entre ellas la obesidad. Así, la diabetes supone un riesgo cardiovascular mayor en aquellos pacientes que la padecen y una vez se manifiesta es la causa más importante de morbimortalidad.

A lo largo de los últimos años, hemos tenido un cambio en el paradigma del manejo de esta condición, razón por la cual se ha soslayado el enfoque glucocéntrico para hacer una aproximación integral al riesgo global y los demás factores asociados. Los estudios recientes han aportado una valiosa información de seguridad cardiovascular, pero lo más interesante es que han demostrado que algunos grupos farmacológicos generan un beneficio adicional en la población con condición cardiovascular. Es tan fuerte el impacto de estos medicamentos que se están posesionando como la estrategia de manejo inicial para la diabetes.
Palabras clave: diabetes mellitus, infarto del miocardio, enfermedades cardiovasculares.

\begin{abstract}
Diabetes is a prevalent disease with increasing cases worldwide and related to other conditions such as obesity. This disease imposes a greater cardiovascular risk to patients who suffer from it by causing myocardial infarction, cerebrovascular events and peripheral arterial disease, notable causes of morbidity and mortality.

Over the last years we have had a change in the paradigm of the management of this condition, leaving aside a glucocentric approach to make an integral approximation of the global risk and other associated factors. Recent studies have provided valuable information on cardiovascular safety but the most interesting have shown that some pharmacological groups have an additional benefit in this population with a decrease in cardiovascular conditions, with such an impact that they are being taken as the initial management strategy for this disease.
\end{abstract}

Keywords: diabetes mellitus, myocardial infarction, cardiovascular diseases.

\section{Introducción}

La diabetes mellitus se ha convertido en una de las condiciones más prevalentes en las últimas décadas y ha ido de la mano con la pandemia de obesidad. En la actualidad, la diabetes es la séptima causa de mortalidad en Estados Unidos y el mundo, con 5,2 millones de muertes atribuidas de manera directa y una tasa de 82,4 por cada 100000 personas (1). Esta enfermedad es un factor de riesgo mayor de enfermedades cardiovasculares, las cuales contribuyen a que se incrementen las tasas de mortalidad relacionadas. 
Según reportes de la Organización Mundial de la Salud (OMS), todos los tipos de diabetes $(1,2$, gestacional) han aumentado globalmente en las últimas décadas, y los casos han pasado de 108 millones (4,7\%) en 1980 a 425 millones en 2017 (8,5 \%). Se estima que en 2045 habrá 629 millones de personas con diabetes en el mundo (2). Además de estas alarmantes cifras de prevalencia, la enfermedad aún no se diagnostica en 193 millones de personas en el mundo, de las cuales 120 millones se encuentran en Asia y en el oeste del Pacífico. Esta situación se debe a factores relacionados con el acceso a los cuidados de salud y a la evolución silenciosa de la enfermedad (1).

La enfermedad cardiovascular ateroesclerótica (enfermedad coronaria, cerebrovascular, arterial periférica) es la principal causa de morbimortalidad en personas diabéticas (3). Las condiciones comunes que coexisten en pacientes diabéticos (la hipertensión arterial sistémica, dislipidemia) son claros factores de riesgo cardiovascular y el control de estos factores previene o ralentiza la enfermedad aterosclerótica (4).

En 2007, tras la publicación de un metaanálisis que demostró un aumento del riesgo de infarto de miocardio (43\%) y de muerte cardiovascular (5) con el uso de rosiglitazona, las agencias reguladoras de medicamentos consideraron la necesidad de exigir estudios de seguridad cardiovascular, en una población per se de alto riesgo, antes de aprobar una nueva molécula (6). Aunque de manera inicial estos experimentos clínicos buscaron lograr un margen seguro de no inferioridad (en comparación con el placebo), cuando se adicionaron al manejo basal del paciente, los resultados han sido consistentes y han demostrado no solo seguridad sino un beneficio en la salud cardiovascular. Esto ha generado un cambio en el paradigma sobre el manejo de este grupo especial de enfermos.

\section{Morbilidad y mortalidad de la enfermedad cardiovascular en personas diabéticas}

La muerte cardiovascular genera el $44 \%$ de todas las defunciones en personas con diabetes tipo 1 y el $52 \%$ en individuos con diabetes tipo 2 (7). La diabetes tipo 2 incrementa de 2 a 6 veces el riesgo de muerte por etiología cardiovascular, mientras que la prevalencia ajustada a la edad de población norteamericana para enfermedades coronarias es el doble en las personas diabéticas, en comparación con aquellas sin la patología (8).

Las presentaciones iniciales de la enfermedad cardiovascular en la diabetes comúnmente son la enfermedad arterial periférica (16,2 \% o 3 veces más) y la falla cardíaca (14,7\%), seguidas del infarto de miocardio no fatal y la angina. En el San Antonio Heart Study, con 4875 pacientes tratados durante 7-8 años se demostró que los diabéticos tuvieron un incremento significativo de la mortalidad por todas las causas (riesgo relativo [RR]: 2,1; intervalo de confianza [IC], $95 \%$ : 1,3-3,5 en hombres) (RR: 8,5; IC, 95 \%: 2,8-25,2 en mujeres) (9).
El riesgo de falla cardíaca se aumenta en un $40 \%$ en pacientes diabéticos, en comparación con sus controles ajustados para la edad (odds ratio [OR]: 2,8; IC, $95 \%$ : 2,2-3,6) (10). Según la Encuesta Nacional de Salud y Nutrición (National Health and Nutrition Examination Survey, NHANES), el 26,3 \% de los eventos cerebrovasculares se asociaron con diabetes; el riesgo fue 2 veces mayor para eventos isquémicos, así como el $50 \%$ para eventos hemorrágicos (11).

Las tasas de mortalidad posinfarto del miocardio son mayores en diabéticos que en personas sin esta enfermedad, y la tasa de muerte se eleva 4,4 veces en diabéticos sin otros factores de riesgo cardiovascular (12).

\section{La diabetes mellitus como equivalente de riesgo coronario}

Este aspecto es un tema controversial. Previamente, se ha comprobado que los pacientes diabéticos sin historia de infarto de miocardio tienen un riesgo similar de muerte por enfermedad coronaria a aquellas personas sin diabetes pero que han tenido un evento coronario (13). En las mujeres no diabéticas con enfermedad cardíaca isquémica, el riesgo de muerte es de 3,39 (IC, 95 \%: 3,08-3,73) comparado con 3,0 (IC, $95 \%: 2,5-3,6)$ para las diabéticas sin enfermedad coronaria (14).

Sin embargo, se han observado datos opuestos. Un metaanálisis de 12 estudios con 45108 pacientes demostró que los diabéticos presentan actualmente una baja tasa de eventos de coronarios en comparación con las personas que tienen cardiopatía isquémica sin diabetes (15). Los datos de Estados Unidos muestran que el $50 \%$ de las mujeres y el $30 \%$ de los hombres diabéticos están en riesgo bajo e intermedio (16).

En el NHANES, así como en el United Kingdom Prospective Diabetes Study (UKPDS), el riesgo de enfermedad isquémica fue del $12,6 \%$ en mujeres y del $11,6 \%$ en hombres, sin alcanzar el equivalente de riesgo del $20 \%$ a 10 años (17). En otro estudio desarrollado en California, la diabetes tampoco fue un equivalente de riesgo coronario, pues su cociente de riesgo (CR) fue de 1,7 (1,66-1,74) (18).

Entre tanto, en el estudio MESA (Multi-Ethnic Study of Atherosclerosis), la diabetes mostró una variación de 10 veces en el riesgo de enfermedad coronaria, en un rango de 0,4-4 \% por año, mientras que para el riesgo de eventos anuales hubo evidencia adicional en contra de que esta condición fuera un equivalente de riesgo (19).

\section{Escenarios clínicos específicos}

\section{Enfermedad coronaria}

Aproximadamente, el 20-30 \% de los pacientes con enfermedad coronaria tienen diabetes y, de la población restante, hasta al $70 \%$ se les puede diagnosticar diabetes, así como 
intolerancia a los hidratos de carbono, cuando se realiza una curva de tolerancia (20).

\section{Enfermedad arterial periférica}

Se estima que el $30 \%$ de las personas hospitalizadas por enfermedad arterial tienen diabetes (21), con factores como larga duración, control subóptimo, coexistencia de otros factores de riesgo cardiovascular y daño de otros órganos asociados con mayor la prevalencia. En diabéticos, esta condición tiene un diagnóstico en general tardío, relacionado con la neuropatía concomitante, con mayor el riesgo de infección y presentación clínica atípica (22).

\section{Falla cardíaca}

En pruebas clínicas de medicamentos antidiabéticos, el 4-30 \% de los participantes tienen falla cardíaca (23). La enfermedad sin diagnóstico es frecuente en los datos observacionales de cohortes de pacientes diabéticos, las cuales indican que hasta el $28 \%$ de ellos tienen falla cardíaca (25 \% con fracción de eyección del ventrículo izquierdo [FEVI] reducida, y $75 \%$ preservada) (24). Los pacientes diabéticos sin falla cardíaca tienen 2 a 5 veces más riesgo de desarrollar esta condición (25). Incluso el riesgo es del 20-40 \% en prediabéticos (26). La falla cardíaca también aumenta la prevalencia de diabetes y otros estados disglucémicos y se considera un factor de riesgo de diabetes mellitus (27). La prevalencia de diabetes en falla cardíaca muestra datos similares a los mencionados independientemente de la FEVI (28) y el 30-40 \% de los pacientes con falla cardíaca han sido reportados diabéticos o prediabéticos en los estudios clínicos (29). La mortalidad cardiovascular, es $50-90 \%$ mayor en enfermos con las dos condiciones, independiente del fenotipo clínico (30).

Dos estudios han mostrado que los pacientes con falla cardíaca que además son diabéticos y prediabéticos no diagnosticados tienen un mayor riesgo de muerte y de eventos adversos (31). En aquellos con empeoramiento de su falla cardíaca, el diagnóstico nuevo de prediabetes es un factor de riesgo independiente de muerte global y cardiovascular (32).

Las personas con episodios de descompensación la diabetes aumenta el riesgo de muerte intrahospitalaria como a un año de seguimiento y de rehospitalización (33).

\section{Implicaciones de los resultados de beneficio cardiovascular en los ensayos clínicos contemporáneos}

Agonistas del receptor del péptido tipo 1 similar al glucagón (Glucagon Like Peptide-1, GLP-1)

El estudio ELIXA (Evaluation of Lixisenatide in Acute Coronary Syndrome) se realizó en 6068 pacientes, de los cuales todos presentaron una enfermedad cardiovascular previa. El medicamento no fue inferior ni afectó los puntos evaluados (muerte cardiovascular, infarto de miocardio no fatal, enfermedad cerebrovascular no fatal, hospitalización por angina inestable) en los pacientes que sufrieron un evento coronario agudo (34).

En el estudio EXSCEL (Exenatide Study of Cardiovascular Event Lowering), que evaluó la seguridad de la exenatida, se aleatorizaron 14752 pacientes (73\% con historia de enfermedad cardiovascular) y no hubo inferioridad de este medicamento comparado con placebo, con respecto a los resultados primarios evaluados (muerte cardiovascular, infarto de miocardio no fatal, enfermedad cerebrovascular no fatal), con una tendencia numérica, pero no significativa, de disminución del $14 \%$ en el grupo de tratamiento activo (35). Un análisis exploratorio evidenció una reducción en la mortalidad por cualquier causa del $14 \%$. En los pacientes del grupo de enfermedad cardiovascular se observó una reducción relativa del riesgo del $10 \%$ en el resultado primario (CR: 0,9; IC, 95 \%: 0,816-0,999).

En el estudio LEADER (Liraglutide Effect and Action in Diabetes: Evaluation of Cardiovascular Outcome Results), que incluyó a 9340 pacientes con diabetes y alto riesgo cardiovascular (81 \% con enfermedad cardiovascular previa), se aleatorizaron los participantes a tratamiento con liraglutida comparado con placebo. Todos los pacientes tenían una enfermedad de larga evolución y buen control de otros factores. Después de 3,1 años de seguimiento, el medicamento se asoció con una disminución del $13 \%$ en el desenlace compuesto de muerte cardiovascular, infarto no fatal y enfermedad cerebrovascular no fatal. Este fármaco redujo la muerte cardiovascular y la muerte global en el 22 y el $15 \%$ respectivamente (36). Análisis secundarios mostraron tasas menores de desarrollo y progresión de enfermedad renal en el grupo de tratamiento activo.

Entre tanto, el estudio SUSTAIN-6 (Trial to Evaluate Cardiovascular and Other Long-term Outcomes with Semaglutide in Subjects with Type 2 Diabetes), aleatorizó 3297 pacientes diabéticos y de alto riesgo cardiovascular (73\% con historia de enfermedad cardiovascular), a recibir semaglutida o placebo. Después de 2,1 años de seguimiento, el grupo de tratamiento activo mostró una disminución del resultado primario en un $26 \%$ (eventos cardiovasculares mayores [Major Cardiovascular Events (MACE)]: infarto no fatal, enfermedad cardiovascular no fatal, muerte cardiovascular), el cual fue dirigido en gran medida por una reducción del 39 \% de eventos cerebrovasculares no fatales. También se encontró una reducción numérica no significativa de los eventos de infarto de miocardio y una disminución de los resultados secundarios de seguridad renal (37).

En el estudio PIONEER-6 (Peptide Innovation for Early Diabetes Treatment), se aleatorizaron 3182 pacientes (35\% con historia de enfermedad cardiovascular). Se encontró que el fármaco fue no inferior y el grupo de tratamiento tuvo menos riesgo de muerte cardiovascular (12 eventos frente a 30 con 
placebo) y muerte global ( 23 eventos frente a 45 con placebo), en un seguimiento de 16 meses. Sin embargo, en el grupo de tratamiento se presentó un aumento de las complicaciones a nivel de la retina (hemorragia vítrea, ceguera y requerimiento de fotocoagulación o agentes intravítreos) (38).

El estudio Harmony aleatorizó 9463 pacientes, quienes en su totalidad tenían una historia de enfermedad cardiovascular, a recibir albiglutida frente a placebo. Como resultado, se presentó una disminución del $22 \%$ en el compuesto de resultados MACE y se redujo la tasa de infartos en un $25 \%$ (39).

Los metaanálisis de los estudios anteriores muestran que este grupo de medicamentos disminuyen los resultados de MACE en un $12 \%$ (CR: 0,88; IC, $95 \%$ : 0,84-0,94) (40).

En el estudio REWIND (Researching Cardiovascular Events with a Weekly Incretin in Diabetes), 9901 pacientes se aleatorizaron a recibir dulaglutida frente a placebo, con una mediana de seguimiento de 5,4 años. Este trabajo encontró una disminución del $22 \%$ en MACE en el grupo de manejo activo (CR: 0,88; IC, 95 \%: 0,79-0,99) (41).

\section{Inhibidor del cotransportador sodio-glucosa tipo 2}

El estudio EMPA-REG incluyó a 7020 pacientes con diabetes de larga duración (57 \% >10 años) y enfermedad cardiovascular, quienes recibieron empagliflozina o placebo, con un seguimiento de 3,1 años. El medicamento mostró una disminución significativa (14\%) del compuesto de MACE, en comparación con placebo. Este resultado fue dirigido por una reducción en las muertes cardiovasculares (38 \%). La reducción de los eventos fue numérica pero no significativa para los infartos, con un incremento no significativo de los eventos cerebrovasculares (24\%). Además, los análisis secundarios evidenciaron una disminución del 35 \% de la hospitalización por falla cardíaca. La reducción de la muerte global fue del $32 \%$, lo cual se traduce a que es necesario tratar 39 pacientes, durante 3 años, a fin de evitar una muerte (42).

El programa CANVAS (CANagliflozin cardioVascular Assessment Study) integró los datos de los estudios CANVAS y CANVAS-R (CANagliflozin cardioVascular Assessment StudyRenal). Estos trabajos aleatorizaron a 10142 pacientes con diabetes y alto riesgo cardiovascular (65 \% tenían una historia previa de enfermedad cardiovascular), quienes recibieron canagliflozina frente a placebo, con un seguimiento de 3,1 años. La disminución de los MACE fue del $14 \%$ pero no se afectaron los eventos. De manera similar a lo observado en el EMPAREG, se redujeron de manera notoria las hospitalizaciones por falla cardíaca. Los pacientes presentaron un aumento no explicado de fracturas y amputaciones, algo que no fue replicado en otros estudios (43).

El estudio DECLARE-TIMI 58 (Dapagliflozin Effect on Cardiovascular Events-Thrombolysis in Myocardial Infarction 58) reclutó a 17160 pacientes (10 186 no tenían una enfermedad cardiovascular), a fin de evaluar la seguridad de la dapagliflo- zina. En los análisis primarios de eficacia no se demostró una disminución significativa en los MACE. Sin embargo, el resultado combinado de muerte cardiovascular y hospitalizaciones por falla cardíaca sí se redujo de manera significativa (reducción relativa del riesgo [RRR]: $17 \%$ ), lo cual fue dirigido por disminución en las hospitalizaciones de falla cardíaca (27 \%) (44). Este beneficio fue igual tanto en el grupo de enfermedad ateroesclerótica establecida como en el grupo sin evidencia de esta.

El estudio CREDENCE (Canagliflozin and Renal Events in Diabetes with Established Nephropathy Clinical Evaluation) evaluó la seguridad renal de la canagliflozina en 4401 pacientes con diabetes y nefropatía por albuminuria (filtración de 30-90 mL/ $\min / 1,73 \mathrm{~m}^{2}$ ), de los cuales el $65 \%$ tenían una historia de enfermedad cardiovascular establecida. Los pacientes del grupo que recibió el medicamento mostraron una reducción del 30 \% en los resultados renales. El análisis secundario preespecificado que incluía los MACE también encontró una disminución del $20 \%$ (CR: 0,80; IC, 95 \%: 0,67-0,91), mientras que la hospitalización por falla cardíaca fue inferior en un $39 \%$ (CR: 0,61; IC, $95 \%$ : 0,47-0,80), en comparación con placebo (45).

Tal y como puede notarse, por primera vez en la historia de la diabetes se tienen datos de pruebas con beneficios cardiovasculares derivados de medicamentos, los cuales fueron diseñados para el manejo metabólico. Con estos resultados tan claros, las sociedades científicas han empezado a realizar recomendaciones específicas en relación con el manejo: se han comenzado a diseñar algoritmos que parten de la consideración de si el paciente tiene una historia de enfermedad cardiovascular aterosclerótica, o está en muy alto o alto riesgo cardiovascular.

Si es así, e independientemente de que el diagnóstico sea de novo o el paciente ya tenga un tratamiento farmacológico, se da una recomendación a favor del uso de estos medicamentos, lo cual incluye el escenario de una monoterapia sobre la metformina. Entonces, esta recomendación parte de la observación según la cual la mayoría de los pacientes de estos estudios tuvieron un manejo con metformina, tanto para los grupos de tratamiento activo como para el grupo placebo, lo cual hace muy poco probable que el beneficio notado sea por factores externos a la adición de los fármacos usados.

El beneficio en los aspectos cardiovasculares va más allá del control glucémico, pues la disminución promedio de hemoglobina glicosilada en estos estudios ha sido del 0,5 \%, con grupos de pacientes con buen control basal de otros factores de riesgo cardiovascular. Por tanto, se reafirma que es poco probable que este beneficio se haya dado por aspectos externos a la inclusión del tratamiento.

En todo caso, aún se tiene el interrogante con respecto a si estos medicamentos pueden servir para el manejo de personas no diabéticas con enfermedad ateroesclerótica establecida (prevención secundaria) y de muy alto o alto riesgo cardiovascular (prevención primaria). La respuesta a esta pregunta seguramente se resolverá en los próximos años, pues hay en cur- 
so diversos estudios en esta población de pacientes. En efecto, de manera reciente se han publicado los resultados del trabajo DAPA-HF (Dapagliflozin and Prevention of Adverse Outcomes in Heart Failure), realizado en pacientes con falla cardíaca de FEVI reducida, con y sin historia de diabetes mellitus (46). Ante ese panorama, se comprobó un beneficio que fue consistente en toda la población (tanto diabéticos como no diabéticos), el cual consistió en una reducción en la hospitalización por falla cardíaca, así como en muertes de origen cardiovascular (25\%). Esto refuerza las observaciones de los estudios anteriores.

De cualquier forma, hacen falta estudios de costoefectividad, en especial en el escenario de sistemas con recursos limitados como el nuestro. Sin embargo, parecería lógico pensar que, a largo plazo, estos medicamentos que disminuyen las hospitalizaciones, los eventos relacionados con estas hospitalizaciones y las muertes serán costo y socialmente efectivos.

\section{Conclusiones}

La diabetes y la enfermedad cardiovascular son dos condiciones coexistentes e importantes causas de morbilidad y muerte en todo el mundo. Esa situación no es diferente en nuestro país y en nuestra región. Los estudios contemporáneos, los cuales se diseñaron con el objetivo de evaluar la seguridad del tratamiento con los antidiabéticos, han mostrado un beneficio consistente y notorio en este grupo de pacientes, el cual va más allá del control glucémico. Con esto, nos ha surgido la inquietud sobre el papel de estos medicamentos en el manejo del riesgo cardiovascular en pacientes de muy alto y alto riesgo, no diabéticos. Seguramente, estas preguntas se resolverán en un futuro no muy lejano, a partir de los estudios clínicos en curso, cuya probabilidad de generar un cambio en el paradigma de manejo de toda esta población es muy alta.

\section{Referencias}

1. Glovaci D, Fan W, Wong ND. Epidemiology of Diabetes Mellitus and Cardiovascular Disease. Curr Cardiol Rep. 2019;21(4):21.

2. NCD Risk Factor Collaboration (NCD-RisC). Worldwide trends in diabetes since 1980: a pooled analysis of 751 population-based studies with 4.4 million participants. Lancet Lond Engl. 2016;387(10027):1513-30.

3. American Diabetes Association. Economic Costs of Diabetes in the U.S. in 2017. Diabetes Care. 2018;41(5):917-28.

4. American Diabetes Association. 10. Cardiovascular Disease and Risk Management: Standards of Medical Care in Diabetes-2020. Diabetes Care. 2020;43(Suppl 1):S111-34

5. Nissen SE, Wolski K. Effect of rosiglitazone on the risk of myocardial infarction and death from cardiovascular causes. N Engl J Med. 2007;356(24):2457-71.

6. Cosentino F, Grant PJ, Aboyans V, Bailey CJ, Ceriello A, Delgado V, et al. 2019 ESC Guidelines on diabetes, pre-diabetes, and cardiovascular diseases developed in collaboration with the EASD. Eur Heart J. 2020;41(2):255-323.

7. Morrish NJ, Wang SL, Stevens LK, Fuller JH, Keen H. Mortality and causes of death in the WHO Multinational Study of Vascular Disease in Diabetes. Diabetologia. 2001;44(Suppl 2):S14-21.

8. Manson JE, Colditz GA, Stampfer MJ, Willett WC, Krolewski AS, Rosner $\mathrm{B}$, et al. A prospective study of maturity-onset diabetes mellitus and risk of coronary heart disease and stroke in women. Arch Intern Med. 1991;151(6):1141-7.

9. Wei M, Gaskill SP, Haffner SM, Stern MP. Effects of diabetes and level of glycemia on all-cause and cardiovascular mortality. The San Antonio Heart Study. Diabetes Care. 1998;21(7):1167-72.

10. Go AS, Mozaffarian D, Roger VL, Benjamin EJ, Berry JD, Blaha MJ, et al Heart disease and stroke statistics--2014 update: a report from the American Heart Association. Circulation. 2014;129(3):e28-292.

11. Emerging Risk Factors Collaboration, Sarwar N, Gao P, Seshasai SRK, Gobin $\mathrm{R}$, Kaptoge $\mathrm{S}$, et al. Diabetes mellitus, fasting blood glucose concentration, and risk of vascular disease: a collaborative meta-analysis of 102 prospective studies. Lancet Lond Engl. 2010;375(9733):2215-22.

12. Zuanetti G, Latini R, Maggioni AP, Santoro L, Franzosi MG. Influence of diabetes on mortality in acute myocardial infarction: data from the GISSI-2 study. J Am Coll Cardiol. 1993;22(7):1788-94.

13. Juutilainen A, Lehto S, Rönnemaa T, Pyörälä K, Laakso M. Type 2 diabetes as a "coronary heart disease equivalent": an 18-year prospective population-based study in Finnish subjects. Diabetes Care. 2005;28(12):2901-7.

14. Hu FB, Stampfer MJ, Solomon CG, Liu S, Willett WC, Speizer FE, et al. The impact of diabetes mellitus on mortality from all causes and coronary heart disease in women: 20 years of follow-up. Arch Intern Med. 2001;161(14):1717-23.

15. Bulugahapitiya U, Siyambalapitiya S, Sithole J, Idris I. Is diabetes a coronary risk equivalent? Systematic review and meta-analysis. Diabet Med J Br Diabet Assoc. 2009;26(2):142-8.

16. Wong ND, Glovaci D, Wong K, Malik S, Franklin SS, Wygant G, et al. Global cardiovascular disease risk assessment in United States adults with diabetes. Diab Vasc Dis Res. 2012;9(2):146-52.
17. Ford ES. Trends in the risk for coronary heart disease among adults with diagnosed diabetes in the U.S.: findings from the National Health and Nutrition Examination Survey, 1999-2008. Diabetes Care. 2011;34(6):133743.

18. Rana JS, Liu JY, Moffet HH, Jaffe M, Karter AJ. Diabetes and Prior Coronary Heart Disease are Not Necessarily Risk Equivalent for Future Coronary Heart Disease Events. J Gen Intern Med. 2016;31(4):387-93.

19. Malik S, Budoff MJ, Katz R, Blumenthal RS, Bertoni AG, Nasir K, et al. Impact of subclinical atherosclerosis on cardiovascular disease events in individuals with metabolic syndrome and diabetes: the multi-ethnic study of atherosclerosis. Diabetes Care. 2011;34(10):2285-90.

20. Arnold SV, Lipska KJ, Li Y, McGuire DK, Goyal A, Spertus JA, et al. Prevalence of glucose abnormalities among patients presenting with an acute myocardial infarction. Am Heart J. 2014;168(4):466-470.e1.

21. Criqui MH, Aboyans V. Epidemiology of peripheral artery disease. Circ Res. 2015;116(9):1509-26.

22. Uccioli L, Gandini R, Giurato L, Fabiano S, Pampana E, Spallone V, et al. Long term outcomes of diabetic patients with critical limb ischemia followed in a tertiary referral diabetic foot clinic. Diabetes Care. 2010;33(5):977-82.

23. Marso SP, Bain SC, Consoli A, Eliaschewitz FG, Jódar E, Leiter LA, et al Semaglutide and Cardiovascular Outcomes in Patients with Type 2 Diabetes. N Engl J Med. 2016;375(19):1834-44.

24. Boonman-de Winter LJM, Rutten FH, Cramer MJM, Landman MJ, Liem AH, Rutten GEHM, et al. High prevalence of previously unknown heart failure and left ventricular dysfunction in patients with type 2 diabetes. Diabetologia. 2012;55(8):2154-62.

25. Nichols GA, Hillier TA, Erbey JR, Brown JB. Congestive heart failure in type 2 diabetes: prevalence, incidence, and risk factors. Diabetes Care. 2001;24(9):1614-9.

26. Matsushita K, Blecker S, Pazin-Filho A, Bertoni A, Chang PP, Coresh J, et al. The association of hemoglobin a1c with incident heart failure among people without diabetes: the atherosclerosis risk in communities study. Diabetes. 2010;59(8):2020-6.

27. Amato L, Paolisso G, Cacciatore F, Ferrara N, Ferrara P, Canonico S, et al. Congestive heart failure predicts the development of non-insulin-dependent diabetes mellitus in the elderly. The Osservatorio Geriatrico Regione Campania Group. Diabetes Metab. 1997;23(3):213-8.

28. Johansson I, Dahlström U, Edner M, Näsman P, Rydén L, Norhammar A Type 2 diabetes and heart failure: Characteristics and prognosis in preserved, mid-range and reduced ventricular function. Diab Vasc Dis Res. 2018;15(6):494-503.

29. McMurray JJV, Packer M, Desai AS, Gong J, Lefkowitz MP, Rizkala AR, et al Angiotensin-neprilysin inhibition versus enalapril in heart failure. N Engl J Med. 2014;371(11):993-1004.

30. MacDonald MR, Petrie MC, Varyani F, Ostergren J, Michelson EL, Young JB, et al. Impact of diabetes on outcomes in patients with low and preserved ejection fraction heart failure: an analysis of the Candesartan in Heart failure: Assessment of Reduction in Mortality and morbidity (CHARM) programme. Eur Heart J. 2008;29(11):1377-85. 
31. Kristensen SL, Jhund PS, Lee MMY, Køber L, Solomon SD, Granger CB, et al. Prevalence of Prediabetes and Undiagnosed Diabetes in Patients with HF$\mathrm{pEF}$ and HFrEF and Associated Clinical Outcomes. Cardiovasc Drugs Ther. 2017:31(5-6):545-9.

32. Pavlović A, Polovina M, Ristić A, Seferović JP, Veliić I, Simeunović D, et al. Long-term mortality is increased in patients with undetected prediabetes and type-2 diabetes hospitalized for worsening heart failure and reduced ejection fraction. Eur J Prev Cardiol. 2019;26(1):72-82.

33. Targher G, Dauriz M, Laroche C, Temporelli PL, Hassanein M, Seferovic PM, et al. In-hospital and 1-year mortality associated with diabetes in patients with acute heart failure: results from the ESC-HFA Heart Failure LongTerm Registry. Eur J Heart Fail. 2017;19(1):54-65.

34. Pfeffer MA, Claggett B, Diaz R, Dickstein K, Gerstein HC, Køber LV, et al. Lixisenatide in Patients with Type 2 Diabetes and Acute Coronary Syndrome. N Engl J Med. 2015;373(23):2247-57.

35. Holman RR, Bethel MA, Mentz RJ, Thompson VP, Lokhnygina Y, Buse JB, et al. Effects of Once-Weekly Exenatide on Cardiovascular Outcomes in Type 2 Diabetes. N Engl J Med. 2017;377(13):1228-39.

36. Marso SP, Daniels GH, Brown-Frandsen K, Kristensen P, Mann JFE, Nauck MA, et al. Liraglutide and Cardiovascular Outcomes in Type 2 Diabetes. $N$ Engl J Med. 2016;375(4):311-22.

37. Marso SP, Bain SC, Consoli A, Eliaschewitz FG, Jódar E, Leiter LA, et al. Semaglutide and Cardiovascular Outcomes in Patients with Type 2 Diabetes. N Engl J Med. 2016;375(19):1834-44.

38. Husain M, Birkenfeld AL, Donsmark M, Dungan K, Eliaschewitz FG, Franco DR, et al. Oral Semaglutide and Cardiovascular Outcomes in Patients with Type 2 Diabetes. N Engl J Med. 2019;381(9):841-51.

39. Hernandez AF, Green JB, Janmohamed S, D'Agostino RB, Granger $\mathrm{CB}$, Jones NP, et al. Albiglutide and cardiovascular outcomes in patients with type 2 diabetes and cardiovascular disease (Harmony Outcomes): a double-blind, randomised placebo-controlled trial. Lancet Lond Engl. 2018;392(10157):1519-29.

40. Zelniker TA, Wiviott SD, Raz I, Im K, Goodrich EL, Furtado RHM, et al. Comparison of the Effects of Glucagon-Like Peptide Receptor Agonists and Sodium-Glucose Cotransporter 2 Inhibitors for Prevention of Major Adverse Cardiovascular and Renal Outcomes in Type 2 Diabetes Mellitus. Circulation. 2019;139(17):2022-31.

41. Gerstein HC, Colhoun HM, Dagenais GR, Diaz R, Lakshmanan M, Pais P, et al. Dulaglutide and cardiovascular outcomes in type 2 diabetes (REWIND) a double-blind, randomised placebo-controlled trial. Lancet Lond Engl. 2019;394(10193):121-30.

42. Zinman B, Wanner C, Lachin JM, Fitchett D, Bluhmki E, Hantel S, et al. Em pagliflozin, Cardiovascular Outcomes, and Mortality in Type 2 Diabetes. N Engl J Med. 2015;373(22):2117-28.

43. Neal B, Perkovic V, Matthews DR, Mahaffey KW, Fulcher G, Meininger G, et al. Rationale, design and baseline characteristics of the CANagliflozin cardioVascular Assessment Study-Renal (CANVAS-R): A randomized, placebo-controlled trial. Diabetes Obes Metab. 2017;19(3):387-93.

44. Wiviott SD, Raz I, Bonaca MP, Mosenzon O, Kato ET, Cahn A, et al. Dapagliflozin and Cardiovascular Outcomes in Type 2 Diabetes. N Engl J Med. 2019;380(4):347-57.

45. Perkovic V, Jardine MJ, Neal B, Bompoint S, Heerspink HJL, Charytan DM, et al. Canagliflozin and Renal Outcomes in Type 2 Diabetes and Nephropathy. N Engl J Med. 2019;380(24):2295-306.

46. McMurray JJV, Solomon SD, Inzucchi SE, Køber L, Kosiborod MN, Martinez FA, et al. Dapagliflozin in Patients with Heart Failure and Reduced Ejection Fraction. N Engl J Med. 2019;381(21):1995-2008. 


\section{Medtronic}

\section{BOMBA DE INSULINA MINIMED ${ }^{\text {MM }}$ 670G}

CONTECNOLOGÍA SMARTGUARD ${ }^{\text {TM }}$

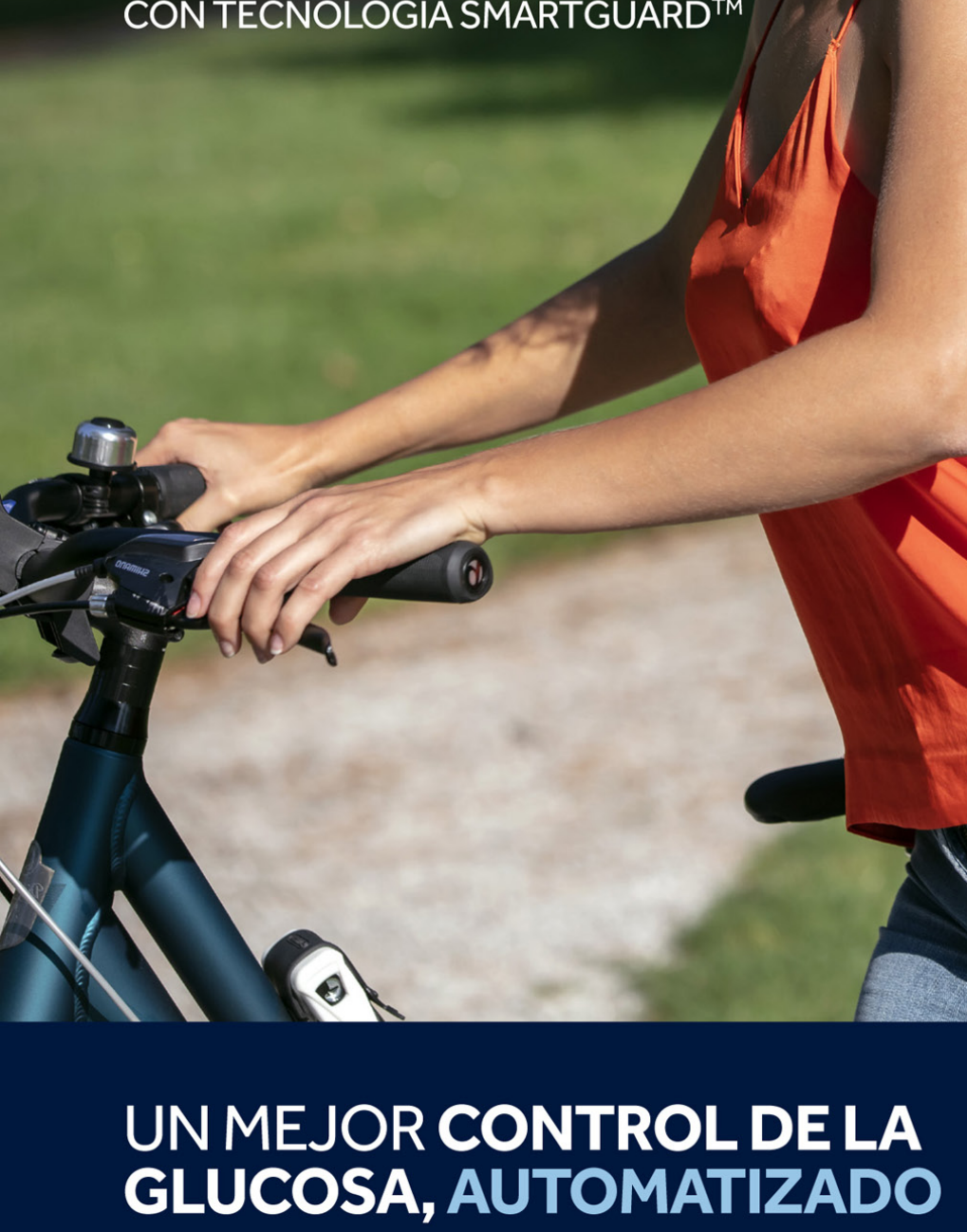

MÁS TIEMPO ENRANGO

Al automatizar la entrega de insulina, el sistema MiniMed ${ }^{\mathrm{TM}} 670 \mathrm{G}$ con tecnología SmartGuard, ayuda a incrementar el tiempo en rango del paciente. ${ }^{1,2}$

\section{MEJOR CONTROL}

¿Cómo sería su día a día si los valores de glucosa de sus pacientes se mantuvieran entre 70 y $180 \mathrm{mg} /$ dL de dia y de noche, automáticamente?*

¡Ahora es posible!

\section{RESULTADOS CLINICOS $^{1,2}$}

- Reducción en HbA1c de 7,4 a 6,9

- Incremento en TIR de $66,7 \%$ a $72,2 \%$

- Cero casos de hipoglucemia grave**

\section{CARELINK SYSTEM}

Gestione y realice un seguimiento del tratamiento con bomba de insulina de sus pacientes, a través del software en línea que genera informes completos e intuitivos.
Ajusta automáticamente la infusión de insulina basal según las lecturas de monitorización continua de glucosa (MCG), adaptándose a las necesidades únicas de insulina de cada paciente.*

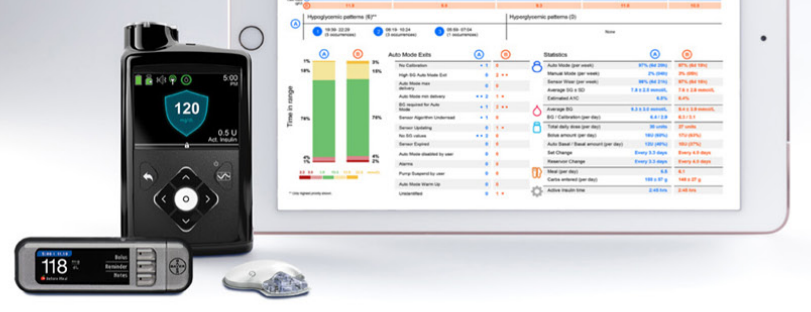

